\title{
Enhancing Non-fouling and Sensitivity of Surface- Enhanced Raman Scattering Substrates for Potent Drug Analysis in Blood Plasma via Fabrication of Flexible Plasmonic Patch
}

Adrianna N. Masterson, Sumon Hati, Greta Ren, Thakshila Liyanage, Nicholas E. Manicke, John V. Goodpaster, and Rajesh Sardar*

Department of Chemistry and Chemical Biology, Indiana University-Purdue University Indianapolis, 402 N. Blackford Street, Indianapolis, Indiana, United States

E-mail: rsardar@iupui.edu

\begin{abstract}
Surface-enhanced Raman scattering (SERS) is an ultrasensitive analytical technique, which is capable of providing high specificity, thus it can be used for toxicological drug assay (detection and quantification). However, SERS-based drug analysis directly in human biofluids requires mitigation of fouling and non-specificity effects that are commonly appeared from unwanted adsorption of endogenous biomolecules present in biofluids (e.g., blood plasma and serum) onto the SERS substrate. Here we report a bottom-up fabrication strategy to prepare ultrasensitive SERS substrates, firstly by functionalizing chemically synthesized gold triangular nanoprisms (Au TNPs) with poly(ethylene glycol)-thiolate in solid-state to avoid protein fouling, and secondly by generating flexible plasmonic patches to enhance SERS sensitivity via the formation of high intensity electromagnetic hot spots. Poly(ethylene glycol)-thiolate-functionalized Au TNPs in the form of flexible plasmonic patches show two-fold improved signal-to-noise ratio in comparison to triethylamine-passivated Au TNPs. Furthermore, the plasmonic patches display a SERS enhancement factor of $4.5 \times 10^{7}$. Utilizing the Langmuir adsorption model we determine the adsorption constant of drugs for two different surface ligands and observed that the drug molecules display stronger affinity for poly(ethylene glycol) ligands than triethylamine. Our density functional theory calculations unequivocally support the interaction between drug molecules and poly(ethylene glycol) moieties. Furthermore, the universality of the plasmonic patch for SERSbased drug detection is demonstrated for cocaine, JWH-018, and opioids (fentanyl, despropionyl fentanyl, and heroin) and binary mixture (trace amount of fentanyl in heroin) analysis. We demonstrate that applicability of flexible plasmonic patches for the selective assay of fentanyl at picogram/milliliter concentration levels from drug-of-abuse patients' blood plasma. The fentanyl concentration determined in the patients' blood plasma from SERS analysis is in excellent agreement with the values determined by paper spray ionization mass spectrometry technique. We believe that the flexible plasmonic patch fabrication strategy would be widely applicable to any plasmonic nanostructures for SERS-based chemical sensing for clinical toxicology and therapeutic drug monitoring.
\end{abstract}

This is the author's manuscript of the article published in final edited form as: 


\section{INTRODUCTION}

Since its discovery in 1974, surface-enhanced Raman scattering (SERS) is one of the most sensitive and emerging spectroscopic techniques currently available for label-free detection of analytes at a very high specificity. ${ }^{1-5}$ The specificity of SERS technique, along with its nondestructive aptitude for analytical assays, can be adapted to numerous detection targets such as small and large biomolecules, microorganisms, and therapeutic and potent drugs. ${ }^{6-11}$ In the development of SERS substrates utilizing plasmonic nanostructures for the analyte detection, two important factors need serious consideration: (1) Generation of a large number of electromagnetic hot spots. It is reported that a near-field plasmonic coupling between metallic nanostructures with sharp tips such as nanostars and triangular nanoprisms provides significantly higher SERS enhancements compared to spherical nanoparticles and nanorods. ${ }^{5,12-15}$ Furthermore, SERS enhancement drastically decreases as analyte molecules move too far from the SERS substrates. ${ }^{2,16}$ (2) Avoid "fouling" which is characterized as competing adsorption of analyte molecules reaching to the electromagnetic hot spots. ${ }^{10}$ The most common approach in SERSbased chemical sensing is to drop-cast the analyte solution onto the SERS substrates. This nonspecific adsorption of analytes onto a SERS-active surface only works for pure analytes. Successful implementation of SERS to chemical diagnostic and forensic toxicology require detection of analytes in blood plasma, which contains plasma proteins. The proteins and analyte compete to reach electromagnetic hot spots via diffusion which results in an increase in background noise that eventually reduces the sensitivity and specificity of the assay.

To improve the SERS-based analyte detection in real-world toxicological samples, it is therefore a paramount importance to design and fabricate SERS substrates that resist nonspecific fouling and produce a larger number of highly intense electromagnetic hot spots. Although various zwitterionic surfaces have been reported to modify the surface of metallic nanostructures for SERS-based chemical and biological analyte detection, however, they required complicated surface modification approaches. ${ }^{10,17-18}$ Herein, we report a unique but simple SERS substrate fabrication strategy that meets both of the above-mentioned requirements to achieve high sensitivity and specificity, while mitigating fouling effects. We used gold triangular nanoprisms (Au TNPS) as plasmonic nanostructures in the SERS substrate fabrication. We adopted a two-step fabrication strategy: Firstly, functionalize Au TNPs with poly(ethylene glycol) thiolate (PEG-S-) to avoid non-specific adsorption of endogenous biomolecules and thus provides excellent nonfouling effects. Secondly, use transparent adhesive tape as a flexible substrate to immobilize PEG-S-functionalized Au TNPs to create large number of electromagnetic hot spots. Together, our fabrication strategy produces "self-assembled" monolayers of PEG-S-functionalized Au TNPs 
onto a transparent and flexible adhesive tape support, which we refer to as "plasmonic patch" for the SERS-based drug analysis.

Many flexible substrates have been developed for SERS-based analyte detection, except for toxicology drug screening. ${ }^{19}$ In most cases, the plasmonic nanostructures were either embedded inside the transparent polydimethylsiloxane (PDMS) film or deposited onto polymer nanofiber films via electrospinning methods. ${ }^{19-23}$ However, both methods displayed some serious drawbacks. For example, analyte molecules do not access the hot spots in the PDMS film, resulting in low sensitivity. To increase sensitivity and enhance the SERS enhancement factor $(\mathrm{EF})$, a separate metal $(\mathrm{Ag}, \mathrm{Au})$ layer is required. Together, this makes the sensing process more complicated. In contrast, electrospinning methods assemble nanostructures onto the surface, but the optically nontransparent nature of polymer fiber films makes the SERS analysis difficult. In this context, adhesive tapes have been used as supporting substrates to fabricate SERS sensors. ${ }^{19}$ Commercially available adhesive tapes are cheap, together making the fabrication process inexpensive. Furthermore, adhesive tapes do not interfere with the SERS effects in the analyte detection, and the extensive roughness of tapes facilitate random organization of nanostructures leading to the formation of large and high intensity hot spots. ${ }^{24}$ Despite the tremendous promises, adhesive tape-based SERS substrate preparation for toxicological drug analysis has yet to be demonstrated. Finally, the colloidal nanostructure previously used in flexible substrate fabrication for SERS-based analyte detection contains surface passivating ligands such as citrate or cetyltrimethylammonium, which are incapable of avoiding fouling effects.

In the present work, plasmonic patches for SERS-based potent drug analysis show much improved non-fouling effects when fentanyl is characterized in diluted blood plasma in comparison to adhesive tape containing self-assembled monolayer of triethylamine-passivated Au TNPs. We determined the fouling effects by characterizing the signal-to-noise (S/N) ratio of SERS spectra of the analyte. We believe this is due to highly specific interaction between drug molecules and poly(ethylene glycol) moiety. Our argument is further supported by time-dependent density functional theory (TDDFT) calculations. Additionally, we determine a large SERS EF of plasmonic patches $\left(4.5 \times 10^{7}\right)$ that we believe to arise due to the formation of "accidental hot spots" from the roughened tape surface. We demonstrate that plasmonic patches can be successfully used for the detection of other potent drugs such as 4-anilino- $\mathrm{N}$-phenethyl-piperidine, cocaine, heroin, and JWH-018, and binary mixture analysis (trace amount of fentanyl in heroin). Finally, as a proof-ofconcept, we analyzed 10 emergency department patients' (drug-of-abuse, DOA) plasma samples to detect and quantify fentanyl and demonstrate the real-life application of our newly fabricated 
SERS substrates in forensic toxicology. The data are in excellent agreement with the concentration values independently determined by paper-spray mass spectrometry.

\section{Experimental Section}

Chemicals. Chloro(triethylphosphine) gold (I) (Et $3 \mathrm{PAuCl}, 97 \%)$, poly(methylhydrosiloxane) (PMHS, $\left.M_{n}=1700-3300\right)$, triethylamine (TEA, 98\%), ACS grade acetonitrile $\left(\mathrm{CH}_{3} \mathrm{CN}, 99.9 \%\right)$, (3aminopropyl)-triethoxysilane, (APTES, 94\%), polyethylene glycol (PEG, Mn 2,000), tetrahydrofuran (THF, 99.9\%), sodium hydroxide $(\mathrm{NaOH}, 97 \%)$, 4-toluenesulfonyl chloride (TsCl, $98 \%$ ), dichloromethane (DCM, 99.5\%), and thiourea (99\%) were purchased from Sigma-Aldrich. Ethanol (alcohol 190 proof) was purchased from Decon laboratories. Glass coverslips (Cat. No. 12548C), RBS 35 detergent, and concentrated hydrochloric acid ( $\mathrm{HCl}$, ACS grade) were purchased from Fisher Scientific. A Fisher Scientific Barnstead Nanopure system was utilized to achieve water purity at $18.2 \mathrm{M} \Omega-\mathrm{cm}$ and the same nanopure water was used for all cleaning processes. Separate $1.0 \mathrm{mg} / \mathrm{mL}$ drug solutions of fentanyl, cocaine, heroin and $\mathrm{JWH}-018$ in methanol were purchased from Fisher Scientific. A $1.0 \mathrm{mg} / \mathrm{mL}$ drug solution of 4-anilino-Nphenethyl-piperidine (4-ANPP) in methanol was purchased from Cerilliant. All drug molecules and solutions were obtained and stored through a DEA license maintained by IUPUI. All DOA and NC plasma samples were collected based on the Indiana University Institute Review Board approval (IRB protocol \# 1810003849).

Preparation of Plasmonic Patches and Drug Detection. The preparation of our flexible and adhesive plasmonic patch is described in Figure 1. Au TNPs with $\sim 42 \mathrm{~nm}$ edge lengths were synthesized according to our published procedure (see Supporting Information for additional details) ${ }^{25-26} \mathrm{Au}$ TNPs in acetonitrile were immobilized onto an APTES-functionalized glass substrate through incubation to form a self-assembled layer of TNPs. The Au TNP-bound coverslips were then incubated in a $1.0 \mathrm{mM} \mathrm{PEG}{ }_{60}-\mathrm{SH}$ solution for overnight for the ligand exchange. Next, 3M adhesive tape was placed on the PEG-S-functionalized Au TNP-containing glass substrate, pressed gently with the thumb, and removed at a $90^{\circ}$ angle. This procedure resulted in successful transfer of the self-assembled Au TNPs from the glass to the flexible adhesive substrate, producing the plasmonic patch (Figure 1C). Drug detection and quantification were performed by directly drop-casting on to the SERS plasmonic patch, followed by slow evaporation of solvent at room temperature.

Limit of Detection (LOD) and SERS EF Calculations. LOD Calculations: Calibration curves were developed by plotting average peak intensity vs. logarithm scale of drug concentration in order to investigate non-specific adsorption at a lower concentration range. The calibration curve equations were determined through linear regression on Excel. The limit of detection (LOD) was determined by using a $Z$ value of the blank $(Z=$ mean $+3 \sigma, \sigma=$ standard deviation), which was obtained using two different spots in three independently fabricated sensors (a total of six SERS measurements) and plugging the $Z$ value into the " $Y$ " in the calibration curve equation and obtaining the LOD concentration ("X"). SERS EF calculations: We followed a previously published method to calculate the enhancement factor of our plasmonic patches at the fentanyl $1384 \mathrm{~cm}^{-1}$ Raman peak, using Eq. 1. ${ }^{27} \mathrm{~N}_{\text {SERS }}$ was calculated by using a laser spot diameter of 5 $\mu \mathrm{M}$ and a fentanyl molecule footprint of $0.76 \mathrm{~nm}^{2}{ }^{27}$ 


\section{Results and Discussion}

Fabrication and Characterization of the SERS Plasmonic Patches. Our newly fabricated plasmonic patches contain a flexible and transparent adhesive tape containing a self-assembled layer of plasmonic nanostructures, Au TNPs. The fabrication strategy is schematically shown in Figure 1 and described below: (1) Freshly prepared, TEA-passivated chemically synthesized $\mathrm{Au}$ TNPs are physiosorbed onto APTES-functionalized glass coverslips via solution phase incubation. A weak intermolecular interaction between TNP-bound TEA and APTES allows physisorption. (2) The surface of TNPs is functionalized with PEG60-SH via ligand exchange chemistry. A soft acid (Au)-soft base (S) interaction easily replaces weakly adsorbed amine ligands from the surface of $\mathrm{Au}$ nanostructures. ${ }^{28}$ (3) 3M adhesive tape is placed onto PEG60-S-functionalized Au TNPs, gently pressed, and then removed slowly to transfer TNPs from the glass support onto the flexible adhesive substrate.

We used Au TNPs because of their unique structural and localized surface plasmon resonance (LSPR) properties. Au TNP contains multiple sharp tips and edges that allow a strong electromagnetic field enhancement due to the formation of SERS hot spots. ${ }^{29}$ Strong Au-S bond is expected to provide long-term stability of the plasmonic patch, specifically in human biofluids. Atomically flat surface of TNPs should induce homogeneous ligand packing, resulting in the improvement of non-specific adsorption of biomolecules from human biofluids and thus less fouling effects. ${ }^{10}$ The synthesis of Au TNPs is conducted by a colloidal reduction method of organometallic gold salt by polymethylhydrosiloxane in acetonitrile. ${ }^{26,30-32}$ We used TEA to control the nucleation and growth processes for the formation of TNPs. Figure 2A illustrates an UVvisible absorption spectrum of Au TNPs exhibiting a characteristic LSPR dipole peak at $\sim 795 \mathrm{~nm}$ in acetonitrile. ${ }^{25,33}$ TEM analysis shows the presence of $42 \pm 6 \mathrm{~nm}$ edge-length Au TNPs (Figure 2B). Based on our reported works, the Au TNPs displaying LSPR dipole absorption peak 795 $\mathrm{nm}$ in acetonitrile should have $\sim 8 \mathrm{~nm}$ in thickness. ${ }^{7}$ TEA-passivated Au TNPs attached onto the APTES-functionalized glass substrate display the LSPR dipole extinction peak at $\sim 730 \mathrm{~nm}$ in air. Upon PEG60-S- functionalization and transferring from glass into flexible substrate, the LSPR dipole peak red shifts to $820 \mathrm{~nm}$ (Figure S1). This $90 \mathrm{~nm}$ dipole peak red shift of Au TNPs is a combination of the formation of Au-S bonds, near-field plasmonic coupling, and the change in their local refractive index (thick PEG60 layer and adhesive polymer of 3M tape). Nevertheless, this LSPR peak position is higher in wavelength and thus suitable for the use of $786 \mathrm{~nm}$ laser for the SERS analysis to enhance signal intensity. ${ }^{34}$ As shown in Figure $2 \mathrm{C}, \mathrm{D}$, the plasmonic patch containing TNPS was analyzed by SEM. The image reveals uniform adsorption of TNPs with $2 \%$ surface coverage without the formation of large aggregates. Therefore, the plasmonic patch can 
be considered as self-assembled flexible substrates. Furthermore, TNPs are randomly oriented in their self-assembled organization onto the flexible substrate, which helps in producing accidental hot spots, overall enhancing the electromagnetic field. Importantly, adhesive tape containing an Au TNP monolayer shows bluish color and the configuration of a plasmonic patch can be replicated like a "Band-Aid" that is expected to provide long-term stability during storage (vide infra).

Analytical Figure of Merits of the Plasmonic Patch for SERS-Based Drug Detection. Herein we test the hypotheses that (1) plasmonic patches are expected to provide a higher SERS sensitivity in comparison to PEG60-S-functionalized Au TNPs adsorbed onto glass substrates and (2) the plasmonic patch will provide an improved non-fouling effect in comparison to TEApassivated Au TNPs adsorbed on either glass or flexible adhesive tape in the SERS analysis. To validate our hypotheses, we compare the above-mentioned four different SERS substrates using fentanyl as a model analyte. Figure 3A shows concentration dependence SERS spectra of fentanyl acquired using plasmonic patches. All the characteristic Raman stretches of fentanyl are detected in the spectrum. We used a distinct H-C-N2 stretch at $1384 \mathrm{~cm}^{-1}$ to determine the LOD, which is found to be $8.9 \mathrm{pM}$ (3.0 picogram/milliliter $(\mathrm{pg} / \mathrm{mL})$ ) (Figure 3B). We selected H-C-N2 for our study instead of commonly used C-C-C trigonal benzene Raman stretch of fentanyl $\sim 1000$ $\mathrm{cm}-1$ because most potent drugs contain multiple aromatic rings in their structures. Therefore, it would be difficult to distinguish drugs such as fentanyl and heroin based on characteristic C-C-C Raman stretches. Instead, H-C-N2 stretch of fentanyl at $1384 \mathrm{~cm}^{-1}$ is distinctly unique and not present in other drugs, thus we expect a much-improved specificity while analyzing drug mixtures, as discussed later in this article. The LOD is more than 50-fold higher than when PEG60-Sfunctionalized Au TNPs adsorbed onto glass substrates was used for SERS-based fentanyl analysis (LOD 594.4 pM, 200 pg/mL), Figure S2A,B. We further determined the LODs for two additional SERS substrates, TEA-passivated Au TNPs adsorbed onto a flexible adhesive substrate $(\mathrm{LOD}=297.2 \mathrm{pM}, 100 \mathrm{pg} / \mathrm{mL})$ and non-flexible glass substrates (LOD $802.4 \mathrm{pM}, 270$ $\mathrm{pg} / \mathrm{mL}$ ), see Figure S3A,B. Concentration dependence SERS spectra of these two substrates are provided in the supporting Information (Figure S4). For the fentanyl detection, the LODs we determined for different plasmonic substrates are at least 100 -fold better than literature reports in which spherical Ag or Ag nanoparticles were used to prepare the SERS substrates. ${ }^{27,}{ }^{35}$ Clearly, the presence of sharp edges and corners of TNPs lead to a strong electromagnetic (EM)-field enhancement in our plasmonic patch that provides the higher sensitivity. In contrast, the EM-field enhancement in spherical nanoparticles is relatively low. ${ }^{29}$ To the best of our knowledge, as 
mentioned above, only spherical metallic nanoparticles were used for the fabrication of SERS substrates for fentanyl detection. In this context, we are the first to demonstrate the successful implementation of anisotropic-shaped nanoparticles for fentanyl analysis using a non-flexible, three-dimensional self-assembled SERS substrate. ${ }^{36}$ Nevertheless, we believe that the flexibility of the adhesive tape brings the TNPs closer to each other, inducing strong near-field interparticle plasmonic coupling between Au TNPs and thus creating high intensity plasmonic hot spots at the nanogap. ${ }^{37}$

We also characterized different SERS substrates (TEA- and PEG60-S-functionalized Au TNPs adsorbed onto glass substrates, and TEA-passivated Au TNPs adsorbed onto a flexible adhesive substrate) by SEM to determine any possible changes in TNP organization during the solvent evaporation steps while drop casting the analyte solution (Figure S5). Interestingly, we observe no change in TEA-passivated TNP assembly before and after addition of analyte solution when they are adsorbed onto the adhesive tape (Figure S5A,B). Since TNPs are embedded inside the adhesive polymer matrix, their movement during the solvent evaporation is highly resistive. In contrast, either TEA-passivated or PEG60-thiolate-functionalized TNPs are chemisorbed onto amine terminated glass substrates via weak Au-N interactions but with an excellent two-dimensional arrangement (Figure S5C-D). Addition of solvent could destroy the two-dimensional self-assembly during the evaporation process. Also, TNPs could migrate onto the solid surface due to a weak Au-N interaction. This is evident from the appearance of aggregated arrangement of TNPs onto glass substrates (Figure S5E-F). Importantly, microscopy analysis of PEG-functionalized Au TNPs adsorbed onto adhesive tape (plasmonic patch) does not show any noticeable change in self-organized assembly of TNPs after drop-casting of the analyte solution (Figure S5G). Together, we believe that the enhanced sensitivity and non-fouling effects of PEG-functionalized Au TNPs onto adhesive tape (plasmonic patch) in the SERS-based drug detection is solely because of specific molecular interactions.

To further understand the substrate effect on SERS sensitivity, we determined and compared SERS EF of plasmonic patch and PEG60-S-functionalized Au TNPs adsorbed onto glass substrates using Eq. 1.

$$
\text { SERS EF }=\left[\frac{I_{S E R S}}{I_{N R}}\right]\left[\frac{N_{N R}}{N_{S E R S}}\right]
$$

Here, ISERS and INR are SERS and normal Raman intensity of fentanyl, respectively. The $\mathrm{N}_{\mathrm{NR}}$ and $\mathrm{N}_{\mathrm{SERS}}$ are number of analyte molecules present in normal Raman and SERS analyses, respectively. Considering fentanyl resides flat onto the surface and TNPs cover nearly $2 \%$ of the total substrate surface, we determined SERS EF based on a literature procedure. ${ }^{27}$ We obtain 
SERS EF to be $4.5 \times 10^{7}$ and $5.2 \times 10^{5}$ for plasmonic patch and PEG60-S-functionalized Au TNPs adsorbed onto glass substrates, respectively. Figure $\mathbf{S 6}$ illustrates a comparison of Raman signal under different experimental conditions used to determine the SERS EF. The results are in agreement with the LOD values mentioned above and also validate our first hypothesis that a higher Raman signal enhancement would be observed when PEG60-S-functionalized TNPs present onto a flexible substrate (plasmonic patch) than non-flexible solid substrate (glass). The SERS EF of $4.5 \times 10^{7}$ we calculated for the plasmonic patch is comparable to Ag-based SERS substrate for fentanyl detection. ${ }^{27}$ It is known that Ag-based substrates provide better SERS EF as compared to Au because of the low optical loss and enhanced LSPR properties. ${ }^{38}$ However, $\mathrm{Ag}$ is more prone to oxidation than $\mathrm{Au}$, which makes the latter more desirable in SERS substrate fabrication. Park et al. reported one order magnitude higher SERS EF when a PDMS-embedded Au nanostars, as a flexible substrate, were used for the SERS analysis. ${ }^{21}$ The reported method requires additional $\mathrm{Ag}$ film substrate, as well as an additional $\mathrm{Si}$ wafer platform, to achieve such EF which makes the analysis more complicated. Table $\mathbf{S 1}$ compiles SERS EF for different flexible substrates. Taken together, the simplicity of the fabrication strategy, high SERS EF, and pg/mL LOD make our plasmonic patch highly desirable for toxicology drug analysis.

We further tested the second hypothesis that the plasmonic patches should display low fouling effects compared to TEA-passivated Au TNPs adsorbed on either glass or flexible adhesive tape in the SERS analysis. A common analytical approach to determine the fouling effect is calculating signal-to-noise $(\mathrm{S} / \mathrm{N})$ ratios at the lowest analyte concentration. ${ }^{10}$ The plasmonic patch displays a $\mathrm{S} / \mathrm{N}$ value of 12.5 for fentanyl at $1.0 \mathrm{nM}$ concentration. In contrast, TEA-passivated Au TNPs adsorbed onto a flexible adhesive substrate shows a S/N value of 4.8 under identical experimental conditions. Figure $\mathbf{S 7}$ shows SERS spectra used for S/N calculations. PEGs are commonly used to functionalize nanostructures in sensing application because they reduce non-specific adsorption of endogenous biomolecules from biofluids. ${ }^{39-40}$ Furthermore, the long glycol polymer should be able to wrap around TNPs, leaving no defect sites in the ligand layer where unwanted molecules could reach to the nanostructure surface. Under such conditions, analyte molecules interact with PEG while endogenous biomolecules are repealed by PEGs. In contrast, TEA is a small molecule and would be unable to provide a homogeneous ligand packing leaving vacant sites, which allow molecular diffusion through them, leading to direct adsorption of analytes onto TNP surface. Therefore, we believe that the lack of control over molecular diffusion in TEA-passivated Au TNPs cause adsorption of both fentanyl and plasma proteins on their surface, causing a higher fouling effect than PEG60-S-functionalized TNPs (plasmonic patch), overall producing a lower S/N ratio. One would expect that a shorter 
PEG chain thiolate ligand would improve the SERS sensitivity due the presence of the analyte molecules much closer the surface of a TNP, however, a shorter PEG could increase the fouling effects. Therefore, it would be interesting to determine the optimum PEG-thiolate chain length for TNP surface passivation to increase the sensitivity without compromising the non-fouling effect. This is part of our ongoing investigation.

To examine the interaction of analytes with ligand functionalized TNP surface in the SERS experiments, we further calculated adsorption constant $\left(K_{a d}\right)$ to quantitatively determine the affinity of such interaction. We used the Langmuir adsorption model to determine $K_{a d}$ utilizing the SERS parameters for fentanyl as described below: ${ }^{41-42}$

$$
I_{S}=\frac{I_{s m} K_{a d}[A]}{1+K_{a d}[A]}
$$

Here, $I_{s}$ and $I_{s m}$ are the SERS intensity at the concentration $A$ and at the saturation point of full monolayer coverage, respectively, and $[A]$ is the concentration of adsorbate (fentanyl). The

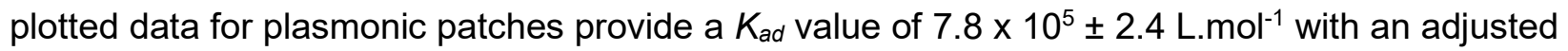
$\mathrm{R}^{2}$ of 0.998 (Figure $3 \mathrm{C}$ ). Interestingly, the $K_{a d}$ value is $2.1 \times 10^{4} \pm 1.3 \mathrm{~L} \mathrm{~mol}^{-1}$ for TEA-passivated $\mathrm{Au}$ TNPs adsorbed onto a flexible adhesive tape (Figure S8). The result is significant and suggests that there is relatively a strong interaction between PEG and fentanyl in comparison to fentanyl and TEA. Therefore, a stronger affinity of fentanyl towards plasmonic patches leads to the greater SERS signal enhancement, and thus the lower is the LOD and fouling effects. ${ }^{41}$ Together, a relatively higher $K_{a d}$ value for plasmonic patch is in agreement with our two hypotheses. The stability/shelf life of the plasmonic patch was also investigated by storing them under normal laboratory condition but covered with a protective layer to prevent prolonged light exposure. As shown in Figure $\mathbf{5 9},<3 \%$ relative standard deviation (RSD) is observed when fentanyl SERS spectra were collected every third day up to a month time.

We further demonstrate that TDDFT calculations can be successfully implemented to support the unique interaction between the plasmonic patch and fentanyl discussed above. We performed TDDFT calculations of fentanyl in which its $\mathrm{N}$ atoms are forming hydrogen bonding with glycol units in aqueous medium. Figure 3D illustrates a comparison of experimental SERS spectrum (red curve) and TDDFT-calculated Raman spectrum (blue curve) of fentanyl. Clearly, $\mathrm{H}-\mathrm{C}-\mathrm{N} 2$ stretch at $1384 \mathrm{~cm}^{-1}$ and the aromatic $\mathrm{C}=\mathrm{C}$ stretch at $1085 \mathrm{~cm}^{-1}$ of fentanyl perfectly match in experimental and theoretical spectra. Furthermore, the ratio of $\mathrm{C}=\mathrm{C}$ to $\mathrm{H}-\mathrm{C}-\mathrm{N} 2$ stretch is almost same for experimental and theoretical data. In contrast, this ratio is different for TDDFT calculated fentanyl Raman spectrum (black curve) alone without any bonding interaction with the PEG in the 
plasmonic patch. Various vibrational modes of fentanyl for both experimental and TDDFTcalculated Raman spectra are provided in the Supporting Information (Table S2). Taken together, the above-mentioned experimental and theoretical results unequivocally prove that the plasmonic patch is the most suitable and efficient SERS substrate for drug detection in human plasma.

\section{Sensing Capabilities of the Plasmonic Patch for SERS-based Various Potent Drugs}

Analysis. An excellent SERS substrate should have the capability to detect any drug family such as coco alkaloids (cocaine), cannabinoids (JWH-018), and opioids (fentanyl, despropionyl fentanyl (4-ANPP), and heroin). Although, literature reports various SERS substrates for the detection of one or two types of drugs in human biofluids (saliva or urine) with high LODs $(>\mathrm{ng} / \mathrm{mL}),{ }^{6,27,41,43-45}$ to the best of our knowledge, currently no SERS substrate exists that could be used to detect such a diverse range of potent drugs in human plasma at $\mathrm{pg} / \mathrm{mL}$ concentrations. The generality of the plasmonic patch for SERS-based drug detection is further demonstrated for the above-mentioned drugs. $1.0 \mathrm{nM}$ concentration drug solution was prepared in diluted human plasma and a total $6.0 \mu \mathrm{L}$ was drop-casted onto the plasmonic patch. Figure 4 shows experimentally acquired SERS spectrum of cocaine, JWH-018, 4-ANPP, and heroin and the comparison with TDDFT-calculated Raman spectra of these drugs. Cocaine displays characteristic aromatic stretch at 1253 and $1600 \mathrm{~cm}^{-1}$, and $\mathrm{C}=\mathrm{O}$ stretch at $1720 \mathrm{~cm}^{-1} .{ }^{45}$ All these vibrational modes are perfectly aligned with the theoretical calculations. Naphthalene $\mathrm{C}=\mathrm{C}$ stretches of JWH-018 appear between $1300-1400 \mathrm{~cm}^{-1}$ along with $\mathrm{C}=\mathrm{O}$ stretch at $1628 \mathrm{~cm}^{-1}$. Another prominent peak of JWH-018 shows up at $775 \mathrm{~cm}^{-1}$ for indole ring stretch. ${ }^{41}$ 4-ANPP shows all the characteristic aromatic peaks as similar to fentanyl. More specifically, a distinct $\mathrm{N}-\mathrm{H}$ stretch is observed at $1035 \mathrm{~cm}^{-1}$ that is not present in fentanyl. The SERS spectrum of heroin is much more complicated than the other drugs. Importantly, we are able to determine $\mathrm{C}=\mathrm{O}$ stretch at $1733 \mathrm{~cm}^{-1}$ and the entire O-C(=O)-C Raman stretch at $1233 \mathrm{~cm}^{-1}$. SERS-based heroin detection has been reported in the literature ${ }^{27,46}$ but a comprehensive list of the Raman stretch for different vibrational modes is still missing. Our thorough TDDFT calculations of heroin show a good match with the experimental spectra, and we are now able to assign most of the experimental Raman vibration modes (Table S6). Therefore, our calculated spectra could be the bedrock to the experimental scientists who are working on forensic science for illicit drug detection (seized drugs and forensic toxicology). A complete list of experimental and theoretical Raman stretch of the above-mentioned drugs is provided in the Supporting Information (Table S3-S6). 
Specificity Test by Binary Mixture Analysis. Fentanyl is commonly used as an adulterant in heroin, and few milligrams of fentanyl may cause an overdose due to its high potency. Therefore, precise quantification of trace quantity fentanyl in binary mixtures is a paramount importance to avoid drug related death and battle with opioid pandemic. Here we show that the plasmonic patches are capable of identifying fentanyl in binary mixture of heroin. Utilizing the SERS intensity ratio between isolated signature peaks for each drug $\left(1384 \mathrm{~cm}^{-1}\right.$ representing the $\mathrm{H}-\mathrm{C}-\mathrm{N} 2$ stretch for fentanyl and $1733 \mathrm{~cm}^{-1}$ representing the $\mathrm{C}=\mathrm{O}$ vibration for heroin) we are able to identify 0.15 wt\% fentanyl in heroin. As shown in Figure S10A, a wide range of weight percentages of fentanyl in heroin (0.15\% to 50\%) were analyzed utilizing SERS. As shown in Figure 5A, heroin-to-fentanyl peak ratio is plotted, and the calibration curve is fitted to a Langmuir isotherm equation following the procedure reported by Haddad et al. ${ }^{27}$ The calibration curve shows a linear increase in peak intensity ratio when the percentage of fentanyl in heroin increases $\left(R^{2}=0.958\right)$, with a gradual plateau around 5\% fentanyl in heroin. Importantly, the lethal concentrations of fentanyl found in binary mixtures are represented in the linear portion of the curve (mg of fentanyl in heroin), proving that the plasmonic patches are capable of detecting trace levels of fentanyl in binary mixtures. More importantly, this is the first example in which below $1 \mathrm{wt} \%$ fentanyl in heroin (as low as 0.15 wt\%) can be detected utilizing SERS than previous reported work by Haddad et al. ${ }^{27}$ In addition, we have also analyzed the SERS spectra obtained for each weight percentage through linear discriminant analysis. Principle component analysis (PCA) was used to generate a Scree Plot, which indicates that the first nine PCs are significant. These PCs represented $93.1 \%$ of the total variance in the data set. Replicates cluster well and subsequent discriminant analysis (DA) using the factor scores from the first nine PCs finds that the different concentrations could be discriminated with $90.9 \%$ accuracy (Figure 5B). Lastly, principle component regression (PCR) generated a model with an $\mathrm{R}^{2}$ of 0.96 and a root mean square error of 7.2 . A plot of the actual versus predicted concentration values is shown in Figure S10B.

Application of the Plasmonic Patch in Forensic Toxicology. Since 1999, an average of 50,000 people die each year from opioid overdose.${ }^{47}$ Emergency department (ED) is the first line of defense to present death by performing initial diagnosis for drug-of-abuse (DOA). ${ }^{48}$ Opioids undergo fast decomposition in the metabolic system and their half-life is few minutes to hours. Therefore, the concentration of undecomposed drugs in human biofluids is expected to be exceedingly low. This is a serious problem for the ED because currently used standard mass spectrometry and ELISA techniques for clinical diagnosis of drugs show low sensitivity. Therefore, there is an unmet need for an ultrasensitive technique to identify drugs in the ED setting in order 
to find appropriate treatment and prevent death. We analyzed 10 patients' plasma samples, which were suspected to contain fentanyl, using the plasmonic patch for SERS-based detection. All the samples show characteristic fentanyl Raman stretch at $1384 \mathrm{~cm}^{-1}$ (Figure 6A). Fentanyl concentration varies between $0.5-12 \mathrm{ng} / \mathrm{mL}$. To further support SERS-based results, we performed PSI-MS analysis of these patient samples. We demonstrated the unique analytical capability of PSI-MS for toxicological drug screen in plasma of DOA patients without performing an extensive sample preparation. ${ }^{49-50}$ As shown in Figure 6B, independently determined concentrations of 10 plasma samples are in excellent agreement between the two techniques. MS-MS spectra of 10 DOA samples showing fentanyl $\mathrm{m} / \mathrm{z}$ value are provided in the supporting Information (Figure S11 and S12A-J). Table S7 provides concentration values determined by SERS and PSI-MS techniques for 10 DOA patient samples. Very recently, Driskell and coworkers reported combined SERS and PSI-MS techniques to analyze seized drugs, however, the method has not been applied to toxicological sample analysis. ${ }^{35}$ As stated earlier, complexity of human biofluids increases fouling effects, and simple paper-based SERS substrate preparation is mostly unlikely to provide high sensitive data. Nevertheless, we present the first example where spectroscopy and spectrometry techniques are used for toxicology drug analysis in real-world samples. We also conducted specificity tests of 10 patient plasma samples $(D O A, n=10)$ with healthy individuals (normal control, NC, $n=10$ ) with a $p$-value of 0.0002 (Figure 6C). As illustrated in Figure 6D, receiving operating characteristic $(R O C)$ analysis shows that the plasmonic patchbased SERS analysis of fentanyl is highly accurate to differentiate between DOA and NC with an area under the curve (AUC) is 0.97 .

\section{Conclusions}

By fabricating a flexible and transparent plasmonic patch, we have demonstrated the highly sensitive, as low as $3.0 \mathrm{pg} / \mathrm{mL}$, detection of potent drugs in human plasma by SERS technique. Moreover, the plasmonic patch mitigates fouling effects commonly caused by human biofluids. Plasmonic patch reported here shows higher SERS EF value $\left(4.5 \times 10^{7}\right)$ compared to the SERS substrate containing PEG60-thilate functionalized Au TNPs adsorbed onto non-flexible glass. Moreover, we have conducted drug adsorption behavior either on PEG60-S- or TEA-passivated Au TNPs adsorbed onto flexible SERS substrates that follows a Langmuir adsorption model, and the drug molecules shows a higher affinity towards PEG surface than TEA. We have successfully detected a range of potent drugs such as cocaine, JWH-018, fentanyl, despropionyl fentanyl, and heroin at $1.0 \mathrm{nM}$ concentrations using the plasmonic patch. The experimental spectra are in good agreement with the TDDFT-calculated spectra and the results suggest molecular level interactions between PEG and drug molecules. Most importantly, through chemometric analysis, 
we have shown excellent selectivity when mixtures of fentanyl in heroin were analyzed using the SERS technique. Using the SERS-based plasmonic patch, we have successfully characterized fentanyl in DOA patients' plasma and the concentration values are in excellent agreement with paper spray ionization-mass spectrometry results determined independently. Finally, ROC analysis presents excellent specificity of identifying fentanyl in DOA comparison to NC samples. Taken together, we believe that the excellent sensitivity and specificity of the plasmonic patch in SERS-based drug detection should be highly advantageous to clinical toxicology because low concentration of undecomposed drugs can be identified. We expect that the developed SERS substrate can also be used in therapeutic drug monitoring and post-mortem toxicology that together will dramatically simplify the "war against drugs."

Supporting Information. Additional experimental details, DFT calculations, SEM images, UVvis extinction and SERS spectra, adsorption isotherm, bar graphs for stability study, chemometric plot, calibration plots for PSI-MS analysis, mass spectra for different patients' samples, and Raman stretch tables of different drugs determined experimentally, and theoretically via DFTcalculations.

Acknowledgements. This project was supported by Award No. 2018-75-CX-0034 awarded by the National Institute of Justice, Office of Justice Programs, the United States Department of Justice. The opinions, findings, and conclusions or recommendations expressed in this publication are those of the authors and do not necessarily reflect those of the Department of Justice.

\section{AUTHOR INFORMATION}

Corresponding Author

${ }^{*}$ Rajesh Sardar; Email: rsardar@iupui.edu

\section{Author Contributions}

R.S conceived the original idea. A.N.M. and T.L conducted all SERS, TEM, and SEM analyses. A.N.M. performed all the SERS data processing, and statistical analysis of patient samples. S.H. performed the DFT calculations and associated data processing. G.R. and N.E.M. performed PSIMS analysis and J.V.G and A.N.M. conducted chemomteric analysis. The manuscript was written from the contribution of all authors, and all authors have given approval to the final version of the manuscript.

Notes. The authors declare no competing financial interest. 


\section{References}

1. Langer, J.; Jimenez de Aberasturi, D.; Aizpurua, J.; Alvarez-Puebla, R. A.; Auguié, B.; Baumberg, J. J.; Bazan, G. C.; Bell, S. E. J.; Boisen, A.; Brolo, A. G.; Choo, J.; Cialla-May, D.; Deckert, V.; Fabris, L.; Faulds, K.; García de Abajo, F. J.; Goodacre, R.; Graham, D.; Haes, A. J.; Haynes, C. L.; Huck, C.; Itoh, T.; Käll, M.; Kneipp, J.; Kotov, N. A.; Kuang, H.; Le Ru, E. C.; Lee, H. K.; Li, J.-F.; Ling, X. Y.; Maier, S. A.; Mayerhöfer, T.; Moskovits, M.; Murakoshi, K.; Nam, J.-M.; Nie, S.; Ozaki, Y.; Pastoriza-Santos, I.; Perez-Juste, J.; Popp, J.; Pucci, A.; Reich, S.; Ren, B.; Schatz, G. C.; Shegai, T.; Schlücker, S.; Tay, L.-L.; Thomas, K. G.; Tian, Z.-Q.; Van Duyne, R. P.; Vo-Dinh, T.; Wang, Y.; Willets, K. A.; Xu, C.; Xu, H.; Xu, Y.; Yamamoto, Y. S.; Zhao, B.; Liz-Marzán, L. M., Present and Future of Surface-Enhanced Raman Scattering. ACS Nano 2020, 14 (1), 28-117.

2. Schlücker, S., Surface-Enhanced Raman Spectroscopy: Concepts and Chemical Applications. Angew. Chem. Int. Ed. 2014, 53 (19), 4756-4795.

3. Stiles, P. L.; Dieringer, J. A.; Shah, N. C.; Van Duyne, R. P., Surface-Enhanced Raman Spectroscopy. Annu. Rev. Anal. Chem. 2008, 1 (1), 601-626.

4. $\quad$ Fleischmann, M.; Hendra, P. J.; McQuillan, A. J., Raman spectra of pyridine adsorbed at a silver electrode. Chem. Phys. Lett. 1974, 26 (2), 163-166.

5. Zong, C.; Xu, M.; Xu, L.-J.; Wei, T.; Ma, X.; Zheng, X.-S.; Hu, R.; Ren, B., Surface-Enhanced Raman Spectroscopy for Bioanalysis: Reliability and Challenges. Chem. Rev. 2018, 118 (10), 4946-4980.

6. Andreou, C.; Hoonejani, M. R.; Barmi, M. R.; Moskovits, M.; Meinhart, C. D., Rapid Detection of Drugs of Abuse in Saliva Using Surface Enhanced Raman Spectroscopy and Microfluidics. ACS Nano 2013, 7 (8), 7157-7164.

7. Masterson, A. N.; Liyanage, T.; Berman, C.; Kaimakliotis, H.; Johnson, M.; Sardar, R., A novel liquid biopsy-based approach for highly specific cancer diagnostics: mitigating false responses in assaying patient plasma-derived circulating microRNAs through combined SERS and plasmon-enhanced fluorescence analyses. Analyst 2020, 145 (12), 4173-4180.

8. Saegmueller, B.; Schwarze, B.; Brehm, G.; Schneider, S., Application of SERS spectroscopy to the identification of (3,4-methylenedioxy)amphetamine in forensic samples utilizing matrix stabilized silver halides. Analyst 2001, 126 (11), 2066-2071.

9. Jarvis, R. M.; Goodacre, R., Characterisation and identification of bacteria using SERS. Chem. Soc. Rev. 2008, 37 (5), 931-936.

10. Sun, F.; Hung, H.-C.; Sinclair, A.; Zhang, P.; Bai, T.; Galvan, D. D.; Jain, P.; Li, B.; Jiang, S.; Yu, Q., Hierarchical zwitterionic modification of a SERS substrate enables real-time drug monitoring in blood plasma. Nat. Commun. 2016, 7 (1), 13437.

11. Nie, S.; Emory, S. R., Probing Single Molecules and Single Nanoparticles by SurfaceEnhanced Raman Scattering. Science 1997, 275 (5303), 1102-1106.

12. Xi, W.; Shrestha, B. K.; Haes, A. J., Promoting Intra- and Intermolecular Interactions in Surface-Enhanced Raman Scattering. Anal. Chem. 2018, 90 (1), 128-143.

13. Liyanage, T.; Rael, A.; Shaffer, S.; Zaidi, S.; Goodpaster, J. V.; Sardar, R., Fabrication of a self-assembled and flexible SERS nanosensor for explosive detection at parts-per-quadrillion levels from fingerprints. Analyst 2018, 143, 2012-2022.

14. Joshi, G. K.; White, S. L.; Johnson, M. A.; Sardar, R.; Jain, P. K., Ultrashort, AngstromScale Decay of Surface-Enhanced Raman Scattering at Hot Spots. J. Phys. Chem. C 2016, 120 (43), 24973-24981. 
15. Le Ru, E. C.; Blackie, E.; Meyer, M.; Etchegoin, P. G., Surface Enhanced Raman Scattering Enhancement Factors: A Comprehensive Study. J. Phys. Chem. C 2007, 111 (37), 13794-13803.

16. Moskovits, M., Surface-enhanced Raman spectroscopy: a brief retrospective. J. Raman Spectrosc. 2005, 36 (6 - 7), 485-496.

17. Panikar, S. S.; Ramírez-García, G.; Sidhik, S.; Lopez-Luke, T.; Rodriguez-Gonzalez, C.; Ciapara, I. H.; Castillo, P. S.; Camacho-Villegas, T.; De la Rosa, E., Ultrasensitive SERS Substrate for Label-Free Therapeutic-Drug Monitoring of Paclitaxel and Cyclophosphamide in Blood Serum. Anal. Chem. 2019, 91 (3), 2100-2111.

18. Panikar, S. S.; Banu, N.; Haramati, J.; Gutierrez-Silerio, G. Y.; Bastidas-Ramirez, B. E.; Tellez-Bañuelos, M. C.; Camacho-Villegas, T. A.; Toro-Arreola, S. d.; De la Rosa, E., Anti-fouling SERS-based immunosensor for point-of-care detection of the B7-H6 tumor biomarker in cervical cancer patient serum. Analytica Chimica Acta 2020, 1138, 110-122.

19. Li, Z.; Huang, X.; Lu, G., Recent developments of flexible and transparent SERS substrates. J. Mater. Chem. C 2020, 8 (12), 3956-3969.

20. Fernández-López, C.; Polavarapu, L.; Solís, D. M.; Taboada, J. M.; Obelleiro, F.; Contreras-Cáceres, R.; Pastoriza-Santos, I.; Pérez-Juste, J., Gold Nanorod-pNIPAM Hybrids with Reversible Plasmon Coupling: Synthesis, Modeling, and SERS Properties. ACS Appl. Mater. Interfaces 2015, 7 (23), 12530-12538.

21. Park, S.; Lee, J.; Ko, H., Transparent and Flexible Surface-Enhanced Raman Scattering (SERS) Sensors Based on Gold Nanostar Arrays Embedded in Silicon Rubber Film. ACS Appl. Mater. Interfaces 2017, 9 (50), 44088-44095.

22. Wen, X.; Li, G.; Zhang, J.; Zhang, Q.; Peng, B.; Wong, L. M.; Wang, S.; Xiong, Q., Transparent free-standing metamaterials and their applications in surface-enhanced Raman scattering. Nanoscale 2014, 6 (1), 132-139.

23. Lu, G.; Li, H.; Zhang, H., Gold-Nanoparticle-Embedded Polydimethylsiloxane Elastomers for Highly Sensitive Raman Detection. Small 2012, 8 (9), 1336-1340.

24. Chen, J.; Huang, Y.; Kannan, P.; Zhang, L.; Lin, Z.; Zhang, J.; Chen, T.; Guo, L., Flexible and Adhesive Surface Enhance Raman Scattering Active Tape for Rapid Detection of Pesticide Residues in Fruits and Vegetables. Anal. Chem. 2016, 88 (4), 2149-2155.

25. Joshi, G. K.; McClory, P. J.; Muhoberac, B. B.; Kumbhar, A.; Smith, K. A.; Sardar, R., Designing Efficient Localized Surface Plasmon Resonance-Based Sensing Platforms: Optimization of Sensor Response by Controlling the Edge Length of Gold Nanoprisms. J. Phys. Chem. C 2012, 116 (39), 20990-21000.

26. Liyanage, T.; Nagaraju, M.; Johnson, M.; Muhoberac, B. B.; Sardar, R., Reversible Tuning of the Plasmoelectric Effect in Noble Metal Nanostructures Through Manipulation of Organic Ligand Energy Levels. Nano Lett. 2020, 20 (1), 192-200.

27. Haddad, A.; Comanescu, M. A.; Green, O.; Kubic, T. A.; Lombardi, J. R., Detection and Quantitation of Trace Fentanyl in Heroin by Surface-Enhanced Raman Spectroscopy. Anal. Chem. 2018, 90 (21), 12678-12685.

28. Sardar, R.; Park, J.-W.; Shumaker-Parry, J. S., Polymer-Induced Synthesis of Stable Gold and Silver Nanoparticles and Subsequent Ligand Exchange in Water. Langmuir 2007, 23 (23), 11883-11889.

29. Hao, E.; Schatz, G. C., Electromagnetic fields around silver nanoparticles and dimers. J. Chem. Phys. 2004, 120 (1), 357-366. 
30. Joshi, G. K.; Deitz-McElyea, S.; Liyanage, T.; Lawrence, K.; Mali, S.; Sardar, R.; Korc, M., Label-Free Nanoplasmonic-Based Short Noncoding RNA Sensing at Attomolar Concentrations Allows for Quantitative and Highly Specific Assay of MicroRNA-10b in Biological Fluids and Circulating Exosomes. ACS Nano 2015, 9 (11), 11075-11089.

31. Joshi, G. K.; McClory, P. J.; Dolai, S.; Sardar, R., Improved localized surface plasmon resonance biosensing sensitivity based on chemically-synthesized gold nanoprisms as plasmonic transducers. J. Mater. Chem. 2012, 22 (3), 923-931.

32. Joshi, G. K.; Blodgett, K. N.; Muhoberac, B. B.; Johnson, M. A.; Smith, K. A.; Sardar, R., Ultrasensitive Photoreversible Molecular Sensors of Azobenzene-Functionalized Plasmonic Nanoantennas. Nano Letters 2014, 14 (2), 532-540.

33. Joshi, G. K.; Smith, K. A.; Johnson, M. A.; Sardar, R., Temperature-Controlled Reversible Localized Surface Plasmon Resonance Response of Polymer-Functionalized Gold Nanoprisms in the Solid State. J. Phys. Chem. C 2013, 117 (49), 26228-26237.

34. McFarland, A. D.; Young, M. A.; Dieringer, J. A.; Van Duyne, R. P., Wavelength-Scanned Surface-Enhanced Raman Excitation Spectroscopy. J. Phys. Chem. B 2005, 109 (22), 1127911285.

35. Burr, D. S.; Fatigante, W. L.; Lartey, J. A.; Jang, W.; Stelmack, A. R.; McClurg, N. W.; Standard, J. M.; Wieland, J. R.; Kim, J.-H.; Mulligan, C. C.; Driskell, J. D., Integrating SERS and PSI-MS with Dual Purpose Plasmonic Paper Substrates for On-Site Illicit Drug Confirmation. Anal. Chem. 2020, 92 (9), 6676-6683.

36. Liyanage, T.; Masterson, A. N.; Hati, S.; Ren, G.; Manicke, N. E.; Rusyniak, D. E.; Sardar, R., Optimization of electromagnetic hot spots in surface-enhanced Raman scattering substrates for an ultrasensitive drug assay of emergency department patients' plasma. Analyst 2020, 145 (23), 7662-7672.

37. Halas, N. J.; Lal, S.; Chang, W.-S.; Link, S.; Nordlander, P., Plasmons in Strongly Coupled Metallic Nanostructures. Chem. Rev. 2011, 111 (6), 3913-3961.

38. Muehlethaler, C.; Leona, M.; Lombardi, J. R., Review of Surface Enhanced Raman Scattering Applications in Forensic Science. Anal. Chem. 2016, 88 (1), 152-169.

39. Liyanage, T.; Masterson, A. N.; Oyem, H. H.; Kaimakliotis, H.; Nguyen, H.; Sardar, R., Plasmoelectronic-Based Ultrasensitive Assay of Tumor Suppressor microRNAs Directly in Patient Plasma: Design of Highly Specific Early Cancer Diagnostic Technology. Anal. Chem. 2019, 91 (3), 1894-1903.

40. Zheng, M.; Davidson, F.; Huang, X., Ethylene Glycol Monolayer Protected Nanoparticles for Eliminating Nonspecific Binding with Biological Molecules. J. Am. Chem. Soc. 2003, 125 (26), 7790-7791.

41. Deriu, C.; Conticello, I.; Mebel, A. M.; McCord, B., Micro Solid Phase Extraction SurfaceEnhanced Raman Spectroscopy ( $\mu$-SPE/SERS) Screening Test for the Detection of the Synthetic Cannabinoid JWH-018 in Oral Fluid. Anal. Chem. 2019, 91 (7), 4780-4789.

42. Izquierdo-Lorenzo, I.; Sanchez-Cortes, S.; Garcia-Ramos, J. V., Adsorption of BetaAdrenergic Agonists Used in Sport Doping on Metal Nanoparticles: A Detection Study Based on Surface-Enhanced Raman Scattering. Langmuir 2010, 26 (18), 14663-14670.

43. Dong, R.; Weng, S.; Yang, L.; Liu, J., Detection and Direct Readout of Drugs in Human Urine Using Dynamic Surface-Enhanced Raman Spectroscopy and Support Vector Machines. Anal. Chem. 2015, 87 (5), 2937-2944. 
44. Han, Z.; Liu, H.; Meng, J.; Yang, L.; Liu, J.; Liu, J., Portable Kit for Identification and Detection of Drugs in Human Urine Using Surface-Enhanced Raman Spectroscopy. Anal. Chem. 2015, 87 (18), 9500-9506.

45. Kline, N. D.; Tripathi, A.; Mirsafavi, R.; Pardoe, I.; Moskovits, M.; Meinhart, C.; Guicheteau, J. A.; Christesen, S. D.; Fountain, A. W., Optimization of Surface-Enhanced Raman Spectroscopy Conditions for Implementation into a Microfluidic Device for Drug Detection. Anal. Chem. 2016, 88 (21), 10513-10522.

46. Fedick, P. W.; Bills, B. J.; Manicke, N. E.; Cooks, R. G., Forensic Sampling and Analysis from a Single Substrate: Surface-Enhanced Raman Spectroscopy Followed by Paper Spray Mass Spectrometry. Anal. Chem. 2017, 89 (20), 10973-10979.

47. $\quad$ https://www.cdc.gov/drugoverdose/index.html, Accessed Sept, 2020. 2020.

48. $\quad$ Lager, P. S.; Attema-de Jonge, M. E.; Gorzeman, M. P.; Kerkvliet, L. E.; Franssen, E. J. F., Clinical value of drugs of abuse point of care testing in an emergency department setting. Tox. Rep. 2018, 5, 12-17.

49. Bills, B. J.; Manicke, N. E., Development of a prototype blood fractionation cartridge for plasma analysis by paper spray mass spectrometry. Clinical Mass Spectrometry 2016, 2 , $18-24$.

50. McKenna, J.; Jett, R.; Shanks, K.; Manicke, N. E., Toxicological Drug Screening using Paper Spray High-Resolution Tandem Mass Spectrometry (HR-MS/MS). Journal of Analytical Toxicology 2018, 42 (5), 300-310. 


\section{LIST of FIGURES}

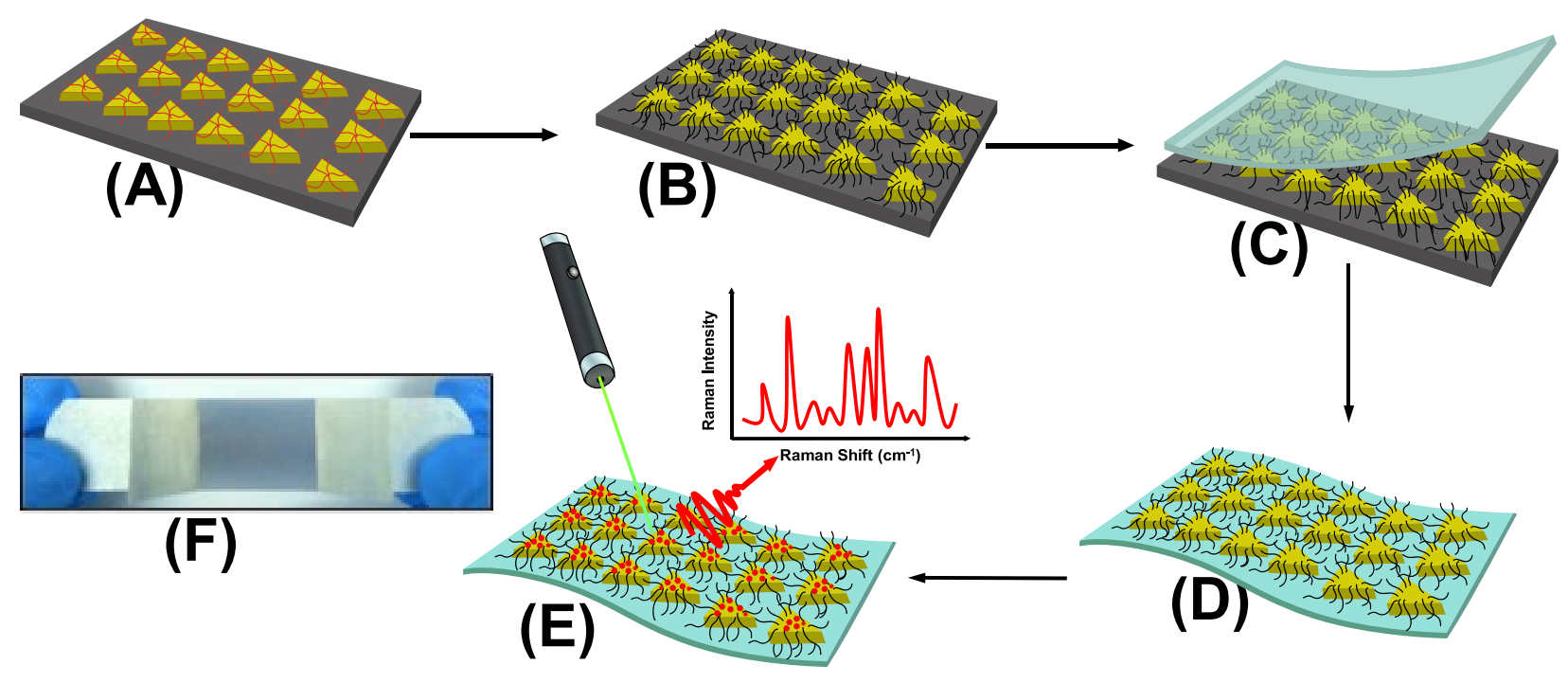

Figure 1. Schematic represent of the fabrication process for our SERS plasmonic patch as follows: Chemically synthesized Au TNPs in acetonitrile are immobilized onto an APTES-functionalized glass substrate through incubation to form a self-assembled layer of TNPs (A), which avoids unwanted aggregation of $\mathrm{Au}$ TNPs and results in reproducible nanosensors fabrication. $\mathrm{Au}$ TNPs are then functionalized with polyethylene glycol-thiolate (B) to enhance specificity. $3 \mathrm{M}$ adhesive tape is placed on the Au TNP-containing glass substrate, pressed gently with the thumb, and removed at a $90^{\circ}$ angle (C) which results in successful lift-off of the Au TNPs from the glass to the tape, producing the plasmonic patch (D). A 6.0 microliter human biofluids can be drop-casted directly onto the nanosensor (D) which results in physisorption of drugs onto TNPs (E). SERS spectra then collected using a Raman spectrometer with 785 $\mathrm{nm}$ laser excitation. (F) The bluish gray area in the photograph is the plasmonic patch and the overall construct resembles with Band-Aid. 

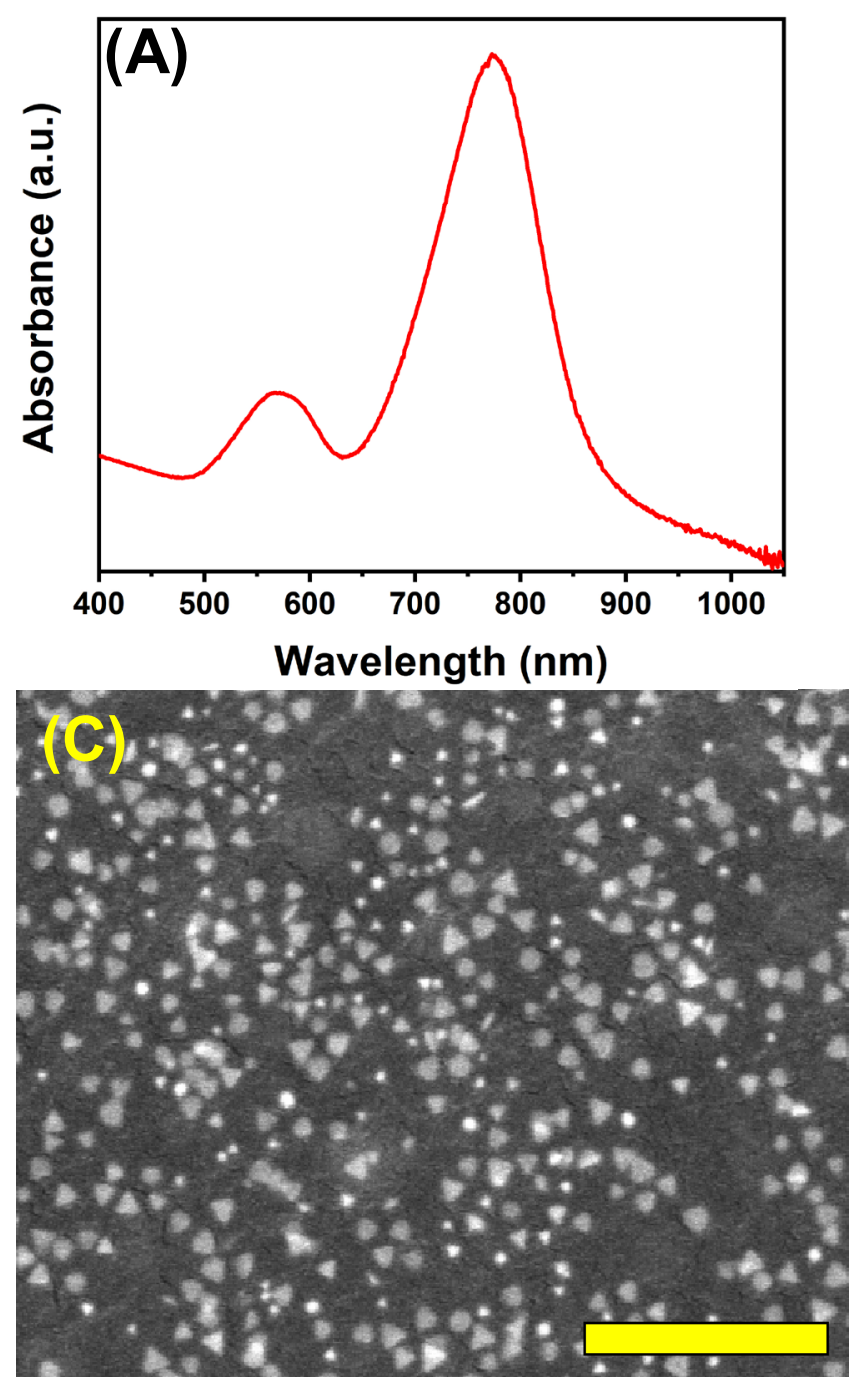
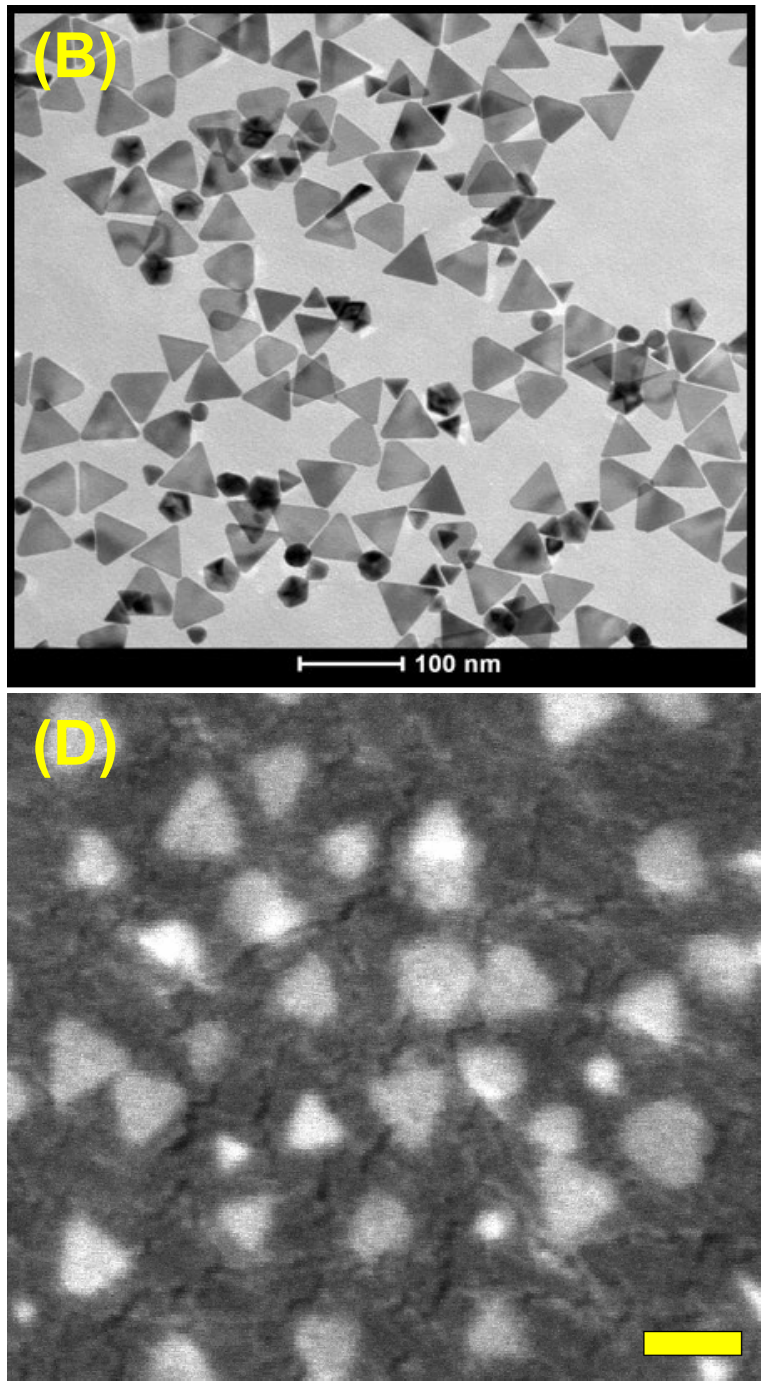

Figure 2. Spectroscopy and microscopy characterizations of Au TNPs and the plasmonic patch. (A) A representative UV-vis absorption spectrum of freshly synthesized TEA-passivated Au TNPs, which display an LSPR dipole peak ( $\lambda$ LSPR) at $795 \mathrm{~nm}$ in acetonitrile. (B) A representative TEM micrograph of Au TNPs. (C) A low magnification SEM image of a plasmonic patch showing self-assembled, PEG-thiolatefunctionalized Au TNPs onto 3M adhesive tape. Scale bar is $500 \mathrm{~nm}$. (D) A high magnification SEM image of a plasmonic patch. No structural deformation of Au TNPs is observed even after adsorption onto the adhesive tape. Scale bar is $50 \mathrm{~nm}$. 

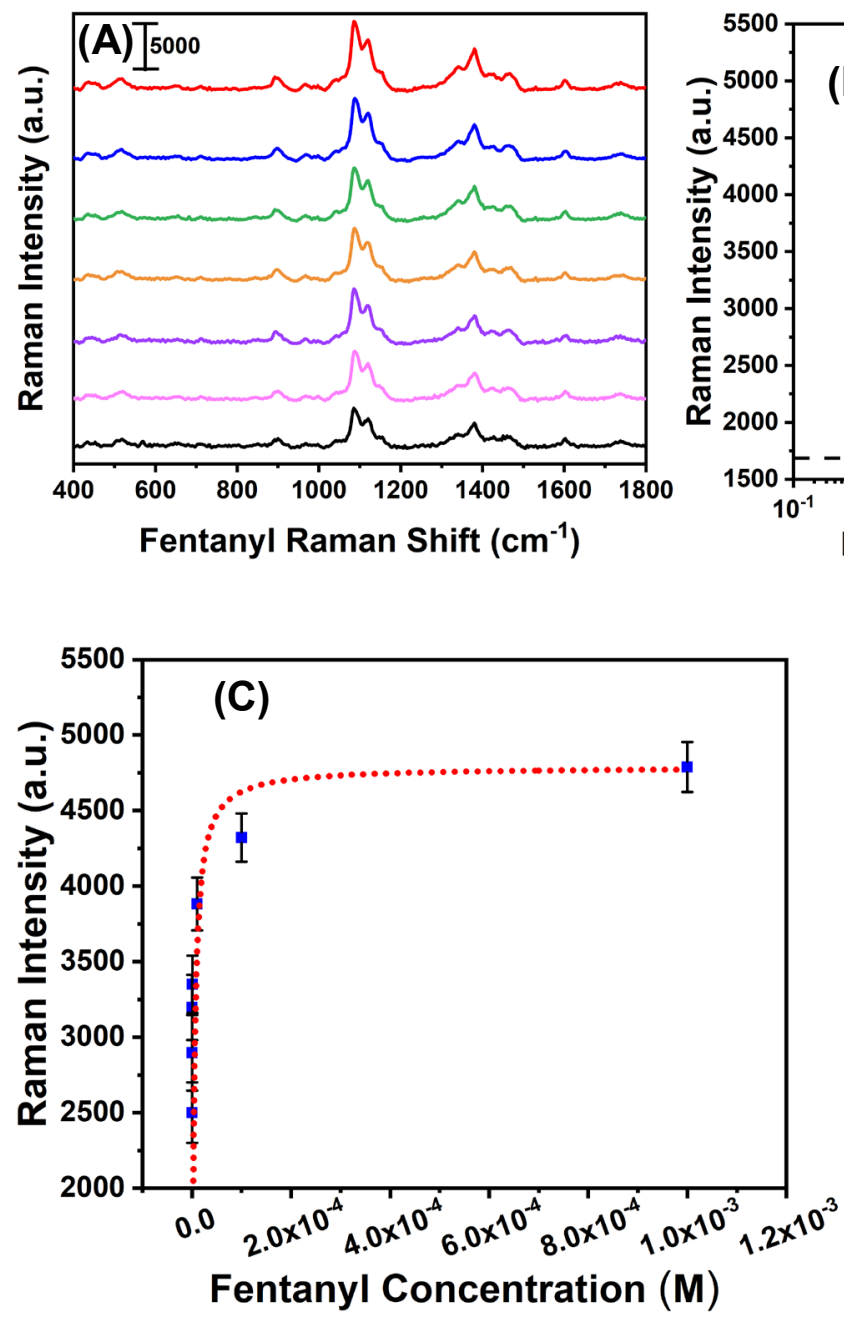
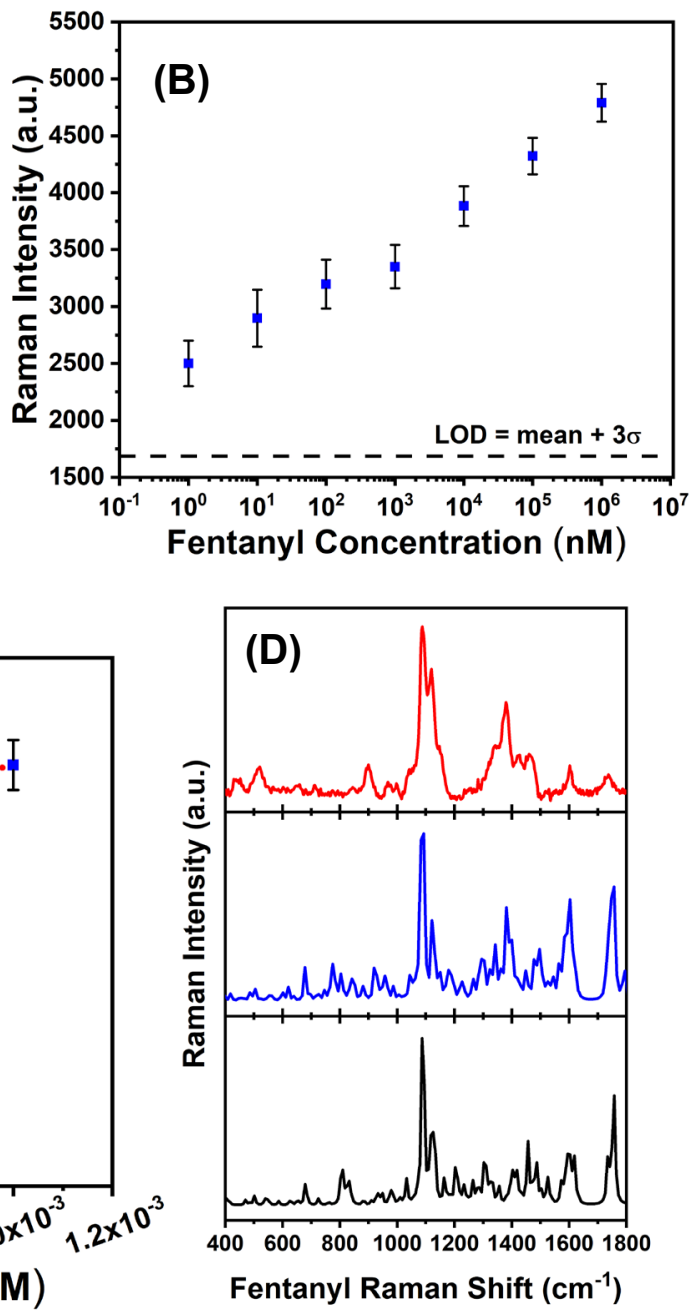

Figure 3. SERS performances of the plasmonic patches. (A) Concentration dependence SERS spectra of fentanyl collected using the plasmonic patch. $(\mathrm{red}=1 \mathrm{mM}$, blue $=100 \mathrm{pM}$, green $=10 \mathrm{pM}$, orange $=1$ $\mathrm{pM}$, purple $=100 \mathrm{nM}$, pink $=10 \mathrm{nM}$, black = $1 \mathrm{nM}$ ). (B) The relationship between fentanyl $\mathrm{H}-\mathrm{C}-\mathrm{N} 2$ stretch as a function of log concentration. Log concentration was used to determine the background signal at the lower concentration range. Each concentration represents two different spots in three independently fabricated sensors (a total of six measurements). (C) Adsorption isotherm of fentanyl on plasmonic patch. The dotted red line is the fit to the Langmuir isotherm model. (D) A comparison of experimentally acquired SERS spectrum of fentanyl (red curve), and theoretically calculated Raman spectra of glycol bound fentanyl (blue curve) and pure fentanyl (black curve). 

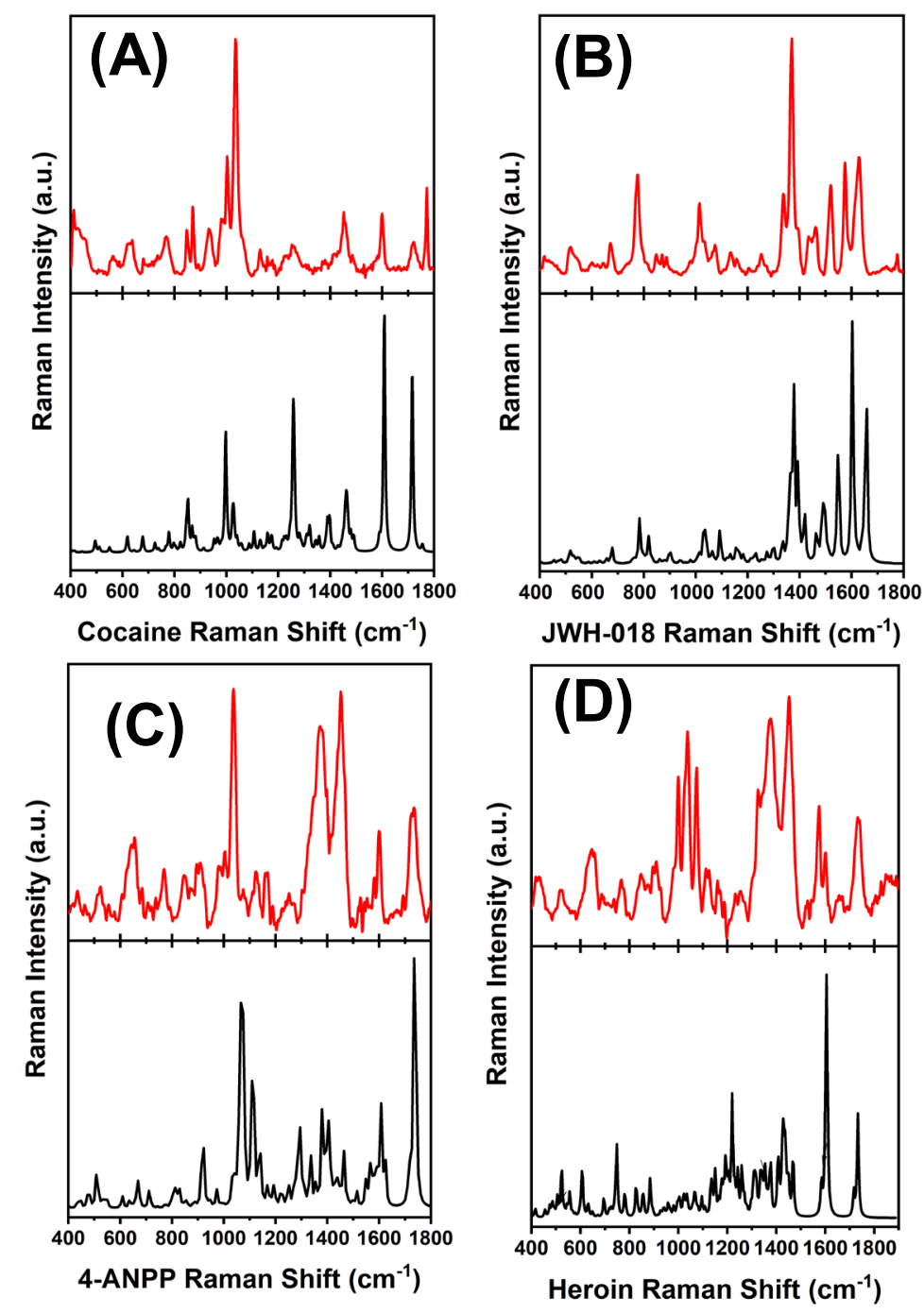

Figure 4. A comparison of experimentally acquired SERS spectrum (red curves) and theoretically calculated Raman spectra of glycol bound fentanyl (black curve) of (A) cocaine, (B) JWH-018, (C) 4-ANPP, and (D) heroin. The concentration of each drug is $1.0 \mathrm{nM}$. 

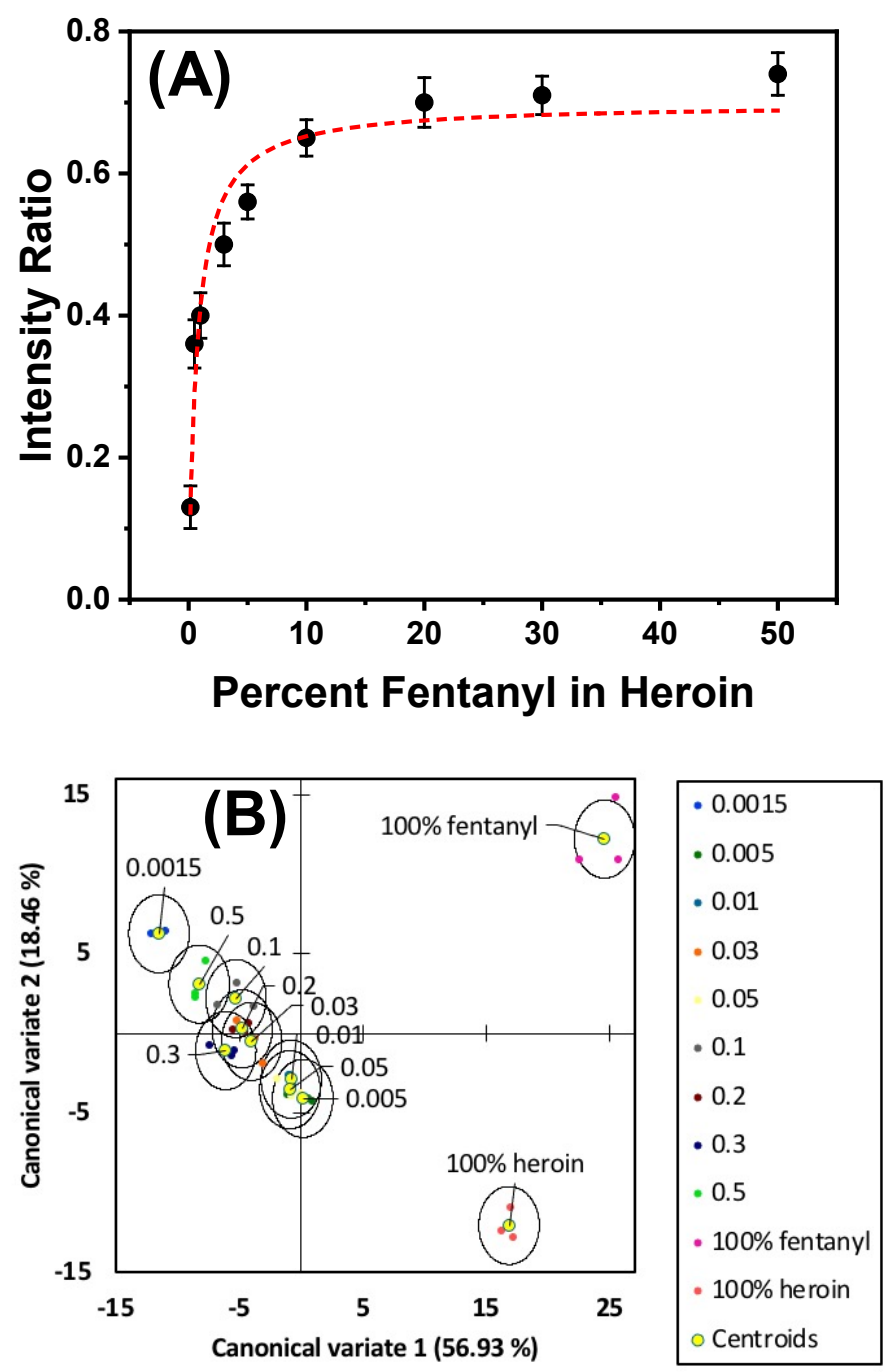

Figure 5. (A) Calibration curve using the peak intensity ratios of fentanyl (H-C-N2 Raman stretch at 1384 $\left.\mathrm{cm}^{-1}\right)$ to heroin $\left(-\mathrm{C}=\mathrm{O}\right.$ Raman stretch at $\left.1733 \mathrm{~cm}^{-1}\right)$ from $0.15-50 \%$. Red dashed line represents calibration curve fitting using the Langmuir isotherm with $R^{2}=0.959$. (B) Scatter plot of the observations and class centroids plotted using the first two canonical variates as determined by discriminant analysis. The overall classification accuracy was $91 \%$. 

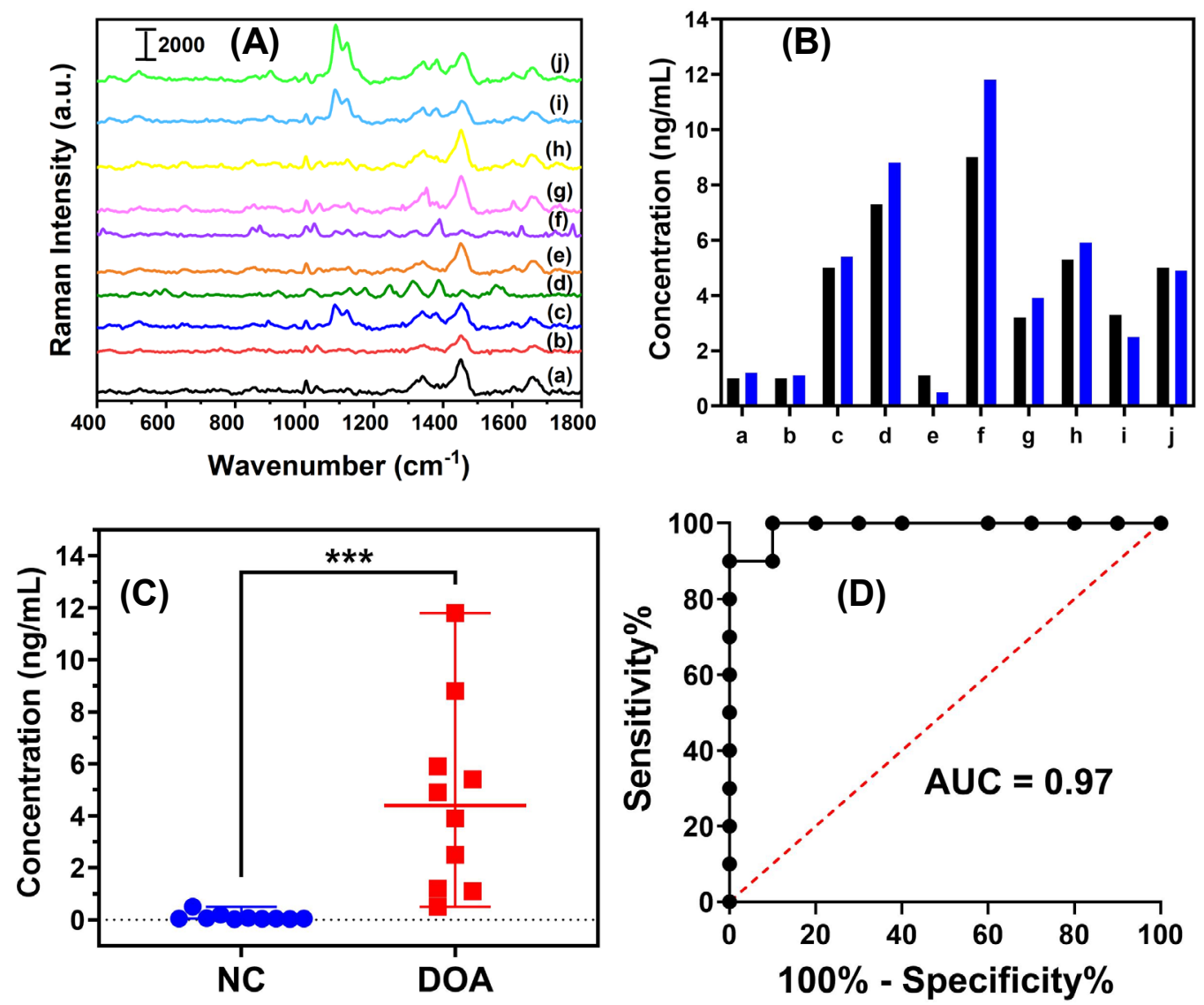

Figure 6. (A) SERS spectra of six microliter-patient plasma diluted to $10 \%(\mathrm{v} / \mathrm{v})$ with RNase free water and then drop-casted onto the plasmonic patch for the analysis. (B) The bar graph representing the concentration value of fentanyl in 10 patient samples where black and blue bars are respective concentrations determined by PSI-MS and SERS techniques. (C) Students t-test results of normal control verses drug-of-abuse (DOA) patient samples for fentanyl. (D) Receiver operating characteristic curve of normal control verses DOA patient samples. ${ }^{* * *} \mathrm{P}<0.0002$ by t-test. 


\section{TOC Graphic}
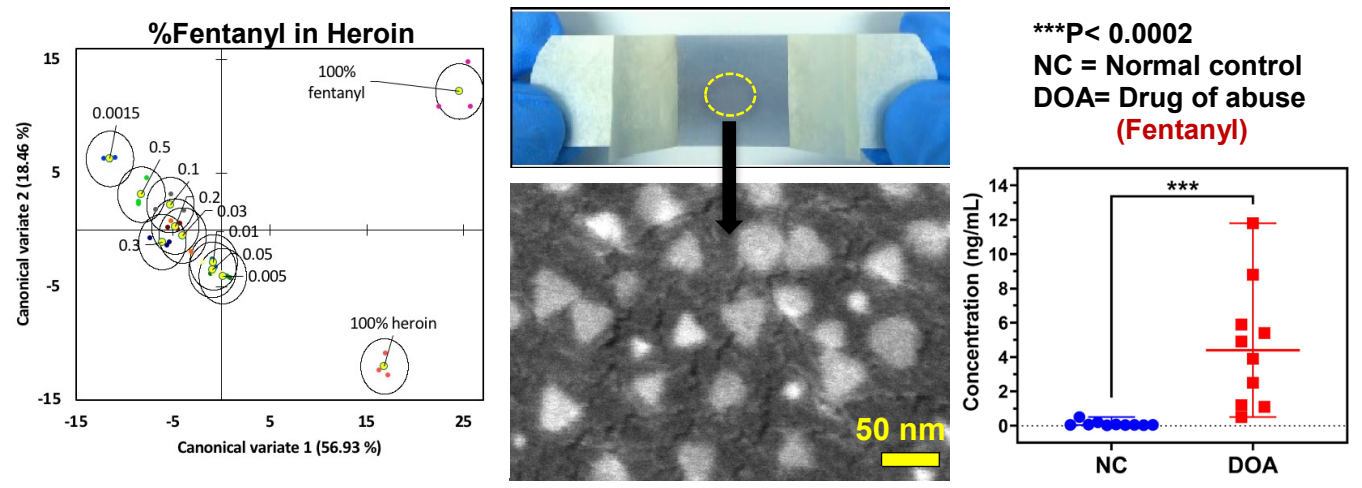